\title{
Improvement in Performance of the VolP over WLAN
}

\author{
Suchi Upadhyay ${ }^{1}$, S.K.Singh ${ }^{2}$ \\ ${ }^{1}$ M.Tech Student, Suresh Gyan Vihar University, Jaipur (Rajasthan), India \\ ${ }^{2}$ M.Tech Co-Ordinator, Suresh Gyan Vihar University, Jaipur, India
}

\begin{abstract}
Wireless VoIP, typically over 802.11 WLAN, is becoming increasingly popular, but even further elevates the challenge of delay and loss reduction. Degradation of speech quality caused by packet delay and loss of voice traffic is still one of the critical technical barriers of the VoIP system. Furthermore, apart from these limitations WLAN will need to support a large number of concurrent VoIP communication since VoIP is spreading rapidly. These motivations led me to do the analysis of VoIP capacity in IEEE 802.11e WLAN and to investigate the methodology to enhance this capacity while maintaining an overall good quality of service.
\end{abstract}

\section{INTRODUCTION}

Voice over Internet protocol (VoIP) is a technology for transmitting voice, video and faxes over the packet switched networks [3]. Using VoIP, voice information is converted into digital packets and sent over the Internet, and converted back into analog signals after reaching the phone receiver at the other end.

VoIP is becoming the reality in business, institutes and home networks. It is serious competitor to the conventional PSTN in terms of cost, efficiency, reliability and quality. It started with making call from computer to computer then progressed to making calls from computer to phone and phone to computer via gateways now phone to phone. It has been accepted widely by business community as using this technology the whole telephony system can be provided over existing IP networks for a fraction of cost of traditional phone. Hence VoIP provides the complete convergence between voice and data networks. However in all these advantages there are some issues as the call is routed over the IP network which is best effort. In conventional telephony the channel is dedicated but in VoIP the call is routed over the IP network hence shares the drawback of packet switched technology i.e. there is no guaranteed quality of service. IP networks were not designed for voice but for data and they are based on the 'Best Effort' principle which means that packets are not guaranteed to be delivered to the receiving station.

Therefore the higher level protocols are responsible for the reliable transmission of data. For data with real time demands, the reliability mechanisms are simply too costly in terms of delay. Additionally an IP network is shared resource utilized by different applications and devices that compete for access to the channel. This competition can lead to bottlenecks, delays or even lost packets which are determined to real time applications. In order to VoIP to work adequately, some requirements have to be fulfilled or in other words there are demands on the Quality of Service from the network. The voice stream must not suffer a delay higher than $150 \mathrm{~ms}$, including processing delays added at the end systems plus the network's latency as this would lessen the interactivity of the conversation. Furthermore, depending on the codec utilized to transform the analog into digital stream of packets a percentage of the lost packets must be kept under a certain minimum, If this is not controlled it may be impossible to reconstruct the voice at the listener in a comprehensive manner. There are mechanisms and technologies that provide the QOS required by VoIP for specific networks bur may do not support them.

\section{Challenges in VoIP over WLAN}

There exist two major challenges for VoWLAN. One challenge is how to increase the system capacity for voice users. Originally designed for data traffic, the WLANs experience bandwidth inefficiency when supporting voice traffic due to the large overhead. Hence, it is essential to enlarge the VoIP capacity supported by WLANs. The other challenge is quality of service $(\mathrm{QoS})$ provisioning for voice users. Voice traffic is sensitive to delay and delay jitter. In current WLANs, VoIP traffic may be interfered by other traffic (e.g., data traffic), resulting in a delay bound violation or large delay variance. Therefore, it is necessary to enhance QoS support capability over WLANs [13], [14], [15].

\section{ANALYSIS AND RESULTS}

DCF provides a best effort service and is not capable of providing differential and prioritization based upon traffic type. It lacks the support for QoS requirements posed by real time traffic like VoIP. PCF mode, with a centralized controller, represents another promising alternative to providing QoS in WLAN. But when the number of the stations in a basic service set (BSS) is large, the polling overheads is high and results in excessive delay. The IEEE 802.11e standard introduces the new coordination functions to support the QoS of VoIP.

\section{Results}

The VoIP capacity in DCF mode and VoIP capacity in IEEE 802.11e WLAN are analyzed on MATLAB and the capacity vs. SF graphs are shown in Fig 1 and Fig 2. 
Maximum number of supported voice calls in DCF/PCF

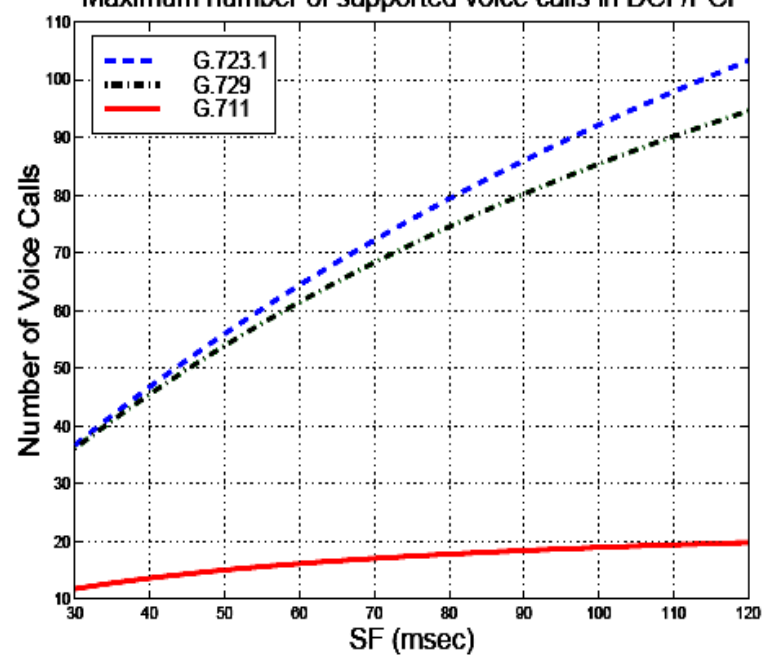

Fig. 1 Maximum Number of Supported Voice Calls in $\mathrm{DCF} / \mathrm{PCF}$

Source [18]

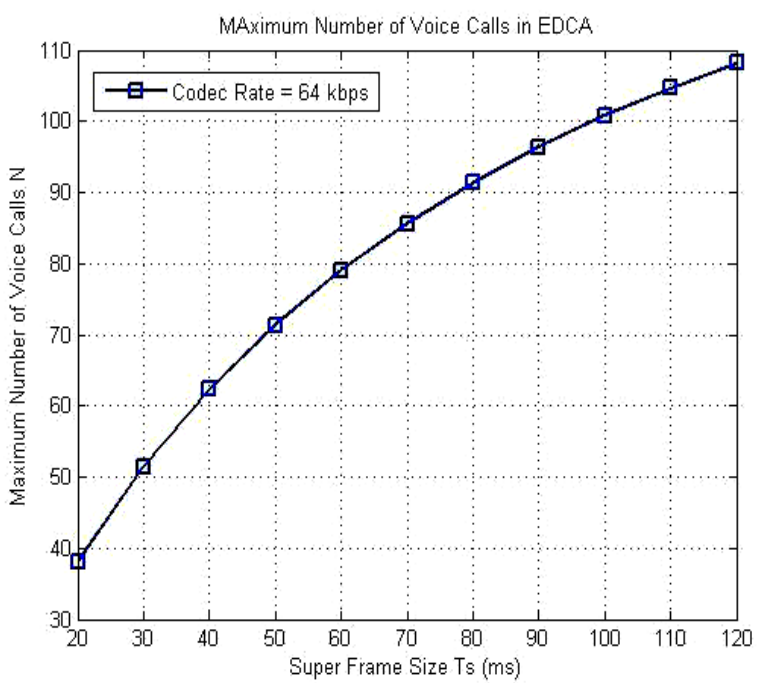

Fig. 2 Maximum Number of Voice Calls in EDCA

The VoIP capacity in an IEEE 802.11 legacy standard using $\mathrm{DCF} / \mathrm{PCF}$ mode of operation (no polling during the $\mathrm{CP}$ ) is being calculated in [10]. The SF size is varied from $30 \mathrm{~ms}$ to $120 \mathrm{~ms}$. The maximum number of VoIP calls with a $120 \mathrm{~ms}$ $\mathrm{SF}$, is 20 calls.

Under EDCA mode, a large increase in the VoIP capacity is achieved. For a $120 \mathrm{~ms} \mathrm{SF}$, the maximum number of VoIP calls is 108. This capacity increase is due to the extension of the CAP during the CP mode of operation.

\section{Results}

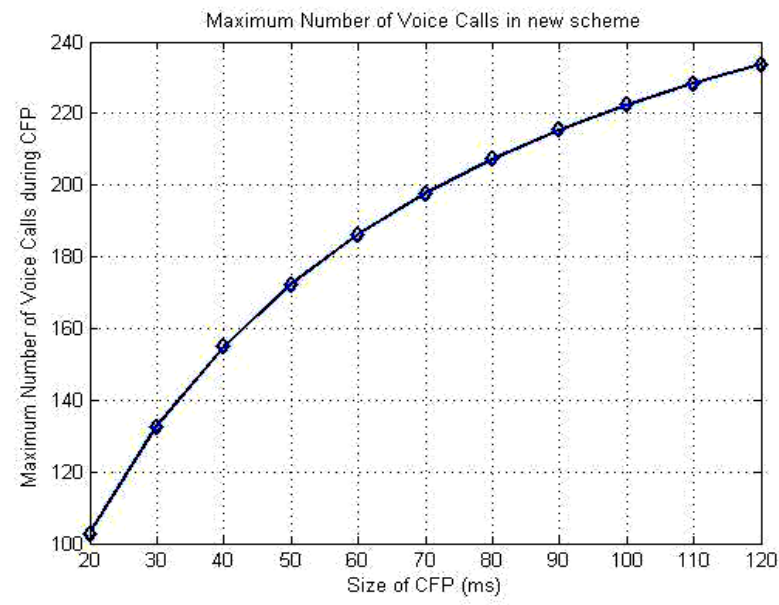

Fig.4 Maximum Number of Voice calls in New Scheme

From the simulation results (see Fig 4.) the number of VoIP calls supported in this scheme for Tsf $=100 \mathrm{~ms}$ is around 235 whereas in existing EDCA scheme the number of voice calls is around 108. Thus this scheme supports more number of calls. Hence provide improved capacity of voice calls.

\section{PROPOSED SCHEME}

In this mechanism, as most multipoll mechanisms, overall polling information is sent only once at the beginning of contention free period (CFP) and each STA retrieve its corresponding polling information. Moreover, each STA only transmits when it receives a number of simple-poll messages (piggybacked with acknowledgment when necessary) that are specified in the multipoll message, the multipoll mechanism maintains the order of STAs' channel access through the number of simple-poll messages a STA should observe before transmission.

\section{PERFORMANCE ANALYSIS AND RESULTS}

By using the multipolling scheme the overheads can be significantly reduced.

For Single Poll scheme

The transmission Time $=328 \mu \mathrm{s}$

Suppose there are $\mathrm{N}$ voice users

Then the total transmission time $=328 * \mathrm{~N}$

For Example $\mathrm{N}=20$

Then Total Transmission Time $=328 * 20=6560 \mu \mathrm{s}$ 
Multipolling Scheme

Size of multipoll frame $=16+7 * \mathrm{~N}$

For $\mathrm{N}=20$

Size of multipoll frame $=16+7 * 20$

$$
=156 \text { bytes }
$$

Total Transmission Time $=192+(156 * 8) / 2$

$$
=816 \mu \mathrm{s}
$$

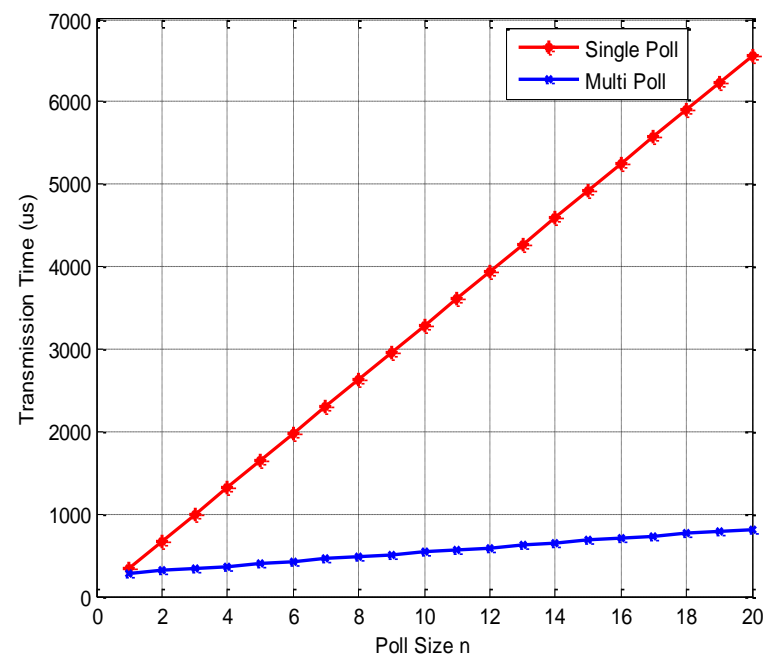

Fig. 5 Total Transmission Time Vs Poll Size for Single Poll and Multi poll

From the Fig 5 it is observed the Total Transmission Time in multipolling scheme is very less as compared to single poll scheme. For the poll size 20, the transmission time for single poll is around $6560 \mu \mathrm{s}$ where as it is $816 \mu \mathrm{s}$ in multipolling scheme.

Now the polling efficiency for both schemes is compared. The following assumptions are made in analysis:

- The PC always performs the initial backoff before sending any polling frame.

- The data frame is transmitted without any acknowledgement. When a STA is polled, the STA either sends a half of frame_num (maximal number of data frames allowed to be transmitted in a TXOP) data frames on average, or sends a null data frame if no data to send.

- There is an equal probability BER (Bit Error Rate) for a bit error to occur due to the channel noise (interference, fading or multipath). Hence, ERRtype (Probability that a type frame is dropped doe to bit errors) can be expressed as $1-(1-B E R)^{\mathrm{I}}$ type .

The polling efficiency is defined by

$$
E=\frac{A v g D}{A v g T}
$$

Where AvgD and AvgT denote the total number of bits in the data frames successfully sent from the polled STAs and the average complete time in time units for a poll, respectively.
The polling efficiency indicates the average uplink data rate during a poll.

\section{- $\quad$ Polling Efficiency of Single poll}

In the single poll, a polled STA contributes data frames if the STA successfully receives a CF-Poll frame and has pending data frames to be successfully transmitted. The polled STA may suffer the frame error due to the interference from external STAs.

Therefore,

Avg $D=(1-a) *$ frame_num $/ 2 * I_{\text {data }} *\left(1-E R R_{\text {data }}\right) *\left(1-E R R_{\text {sin } g l e-p o l l}\right)$

Where different parameters are given in table (see Appendix A)

The polled STA will give a response to the PC after a SIFS period for a successful CF-Poll. If a polled STA does not respond to the PC after a PIFS period for a failed CF-Poll, the PC takes over the channel control and may send the next CFPoll frame. In the HCF, the RTS/CTS frames should be exchanged before the data transmission to prevent the interference from other STAs. Since, the HCF is being substituted for the PCF in the 802.11E, we consider the SinglePoll of the HCF in our analysis. Therefore,

$$
\begin{aligned}
& \text { AvgT }=T_{\text {backoff }}+\left(t_{\text {single-poll }}+t_{\text {PIFS }}\right) * E R R_{\text {single-poll }}+\left[t_{\text {single-poll }}+t_{\text {SIFS }}+\right. \\
& \left.(1-a) * \text { frame_num } 2 *\left(t_{\text {data }}+t_{\text {SIFS }}\right)+a *\left(t_{\text {null }}+t_{\text {SIFS }}\right)\right]^{*}\left(1-E R R_{\text {single-poll })}\right.
\end{aligned}
$$

\section{CONCLUSION}

Due to the limitations of DCF and PCF, the 802.11e defines a single coordination function for QoS data transmission. EDCA supports more number of voice calls as compared to DCF mode. But EDCA also suffers from some limitations. There are large polling overheads. An efficient multipolling scheme is proposed that provides the enhanced polling efficiency and also overcome the hidden node problem.

\section{Future Work}

As we know that the voice is real time traffic and most of the time there is no need of acknowledgement. So when there is no Ack then AP sends the simple polling frame to the STAs (without using piggybacking) and then there will be some extra overheads due to these polling frames. In my future work new scheme will be developed to overcome this problem.

\section{REFERENCES}

[1] IEEE 802.11, 1999 Edition, IEEE Standards for Information Technology, Telecommunication and Information Exchange between Systems Local and Metropolitan Area Network, Specific Requirement, Part 11: "Wireless LAN Medium Access Control (MAC) and Physical Layer (PHY) Specification".

[2] Ray Y.W.Lam, Victor C.M.Leung, "Polling Based Protocols for Packet Voice Transport Over IEEE 802.11 Wireless local Area Networks", IEEE 
Wireless communication, Vol 13, No.1,pg 22-29, February 2006

[3] L. Zhao and C. Fan, "M-PCF modified IEEE 802.11 PCF protocol implementing QoS", Electronic Letters, Vol.38, No.24, pp.1611-1613, Nov. 2002.

[4] P. Brady, "A Model for Generating On-Off Speech Patterns in Two-Way Conversation", Bell Syst. Tech. Journal, vol. 48, no. 7, pp. 2245-2272, Sept. 1969.

[5] WeiWang,Soung Chang Liew and Victor O.K.Li, "Solutions to Performance Problems in VoIP Over a 802.11 Wireless LAN ", IEEE transactions on vehicular technology, vol.54,NO.1,January 2005

[6] A. Kopsel and A. Wolisz, "Voice Transmission in an IEEE 802.11 WLAN Based Access Network", Proc. ACM WOWMOM 01, pp. 23-32, July 2001.
[7] D. P. Hole and F. A. Tobagi, "Capacity of an IEEE 802.11 b wireless LAN supporting VoIP ", IEEE ICC, Vol 1, pp. 196-201, June 2004.

[8] Y.-J. Kim and Y.-J. Suh, "Adaptive Polling MAC Schemes for IEEE 802.11 Wireless LANs Supporting Voice-over-IP (VoIP) Services ", Wireless Commun. Mob. Comp., vol. 4, pp. 903-16, 2004,.

[9] E. Ziouva, T.Antonakopoulos, "Efficient Voice Communication over IEEE802.11 WLANs Using Improved PCF Procedure ",The Third International Network Conference-INC 2002, July 2002.

[10] Takehiro Kawata, Sangho Shin, Andrea G. Forte, "Using Dynamic PCF to improve the Capacity for VoIP Traffic in IEEE 802.11 Networks ",IEEE Communications Society/WCNC, Vol 3, pp. 1589 1595, March 2005. 